\title{
Nucleotide sequences of the genes encoding fructosebisphosphatase and phosphoribulokinase from Xanthobacter flavus H4-14
}

\author{
W. G. Meijer, ${ }^{1} \dagger$ H. G. Enequist, ${ }^{2}$ P. Terpstra ${ }^{2}$ and L. Dijkhuizen ${ }^{1 *}$ \\ ${ }^{1}$ Department of Microbiology, University of Groningen, Kerklaan 30, 9751 NN Haren, The Netherlands \\ ${ }^{2}$ Department of Biochemistry, University of Groningen, Nijenborgh 16, 9747 AG Groningen, The Netherlands
}

(Received 6 July 1990; accepted 9 August 1990)

\begin{abstract}
The genes encoding fructosebisphosphatase and phosphoribulokinase present on a $2.5 \mathrm{~kb}$ SalI fragment from Xanthobacter flavus H4-14 were sequenced. Two large open reading frames (ORFs) were identified, preceded by plausible ribosome-binding sites. The ORFs were transcribed in the same direction and were separated by 39 base pairs. They encoded proteins of 364 and 291 amino acids, with molecular masses of 38739 and 33409 Da, respectively. The ORFs were identified as the genes encoding FBPase and PRK, respectively, on the basis of similarity with FBPase and PRK sequences from other sources.
\end{abstract}

\section{Introduction}

Many organisms, including higher plants, grow autotrophically using the Calvin cycle for $\mathrm{CO}_{2}$ fixation. Three enzymic steps are considered to be unique to this pathway. Phosphoribulokinase (PRK) catalyses the phosphorylation of ribulose 5-phosphate (RuMP) by ATP, yielding ribulose 1,5-bisphosphate (RuBP) and ADP. The RuBP formed is carboxylated by ribulose-1,5bisphosphate carboxylase/oxygenase $(\mathrm{RuBisC} / \mathrm{O})$, leading to the formation of two molecules of 3-phosphoglycerate. RuMP is regenerated by a series of enzymes which, with the exception of sedoheptulosebisphosphatase, also function in other pathways, e.g. during gluconeogenesis. The latter enzyme is bifunctional, also displaying fructosebisphosphatase (FBPase) activity (Tabita, 1988).

The genes encoding FBPase $(c f x F)$ and PRK $(c f x P)$ have been cloned from the autotrophic bacteria Alcaligenes eutrophus, Rhodobacter sphaeroides and Xanthobacter flavus H4-14. In these organisms the $c f x F$ and $c f x P$

† Present address: Department of Microbiology, The Ohio State University, 484 West 12th Avenue, Columbus, OH 43210-1292, USA.

Abbreviations: FBPase, fructosebisphosphatase, F-2,6-P, fructose 2,6-bisphosphate; LB, Luria broth; ORF, open reading frame; PRK, phosphoribulokinase; RuBP, ribulose bisphosphate; $\mathrm{RuBisC} / \mathrm{O}$, ribulose-1,5-bisphosphate carboxylase/oxygenase; RuMP, ribulose monophosphate.

The nucleotide sequence data reported in this paper have been submitted to EMBL and will appear in the EMBL/GenBank/DDBJ Nucleotide Sequence Databases under the accession number X17252. genes are cotranscribed in the order $c f x F-c f x P$ (Gibson \& Tabita, 1987, 1988; Hallenbeck \& Kaplan, 1987; Klintworth et al., 1988; Meijer et al., 1990b). The nucleotide sequences of a number of FBPase-encoding genes have been reported. However, the only Calvin cycle $c f x F$ gene that has been sequenced is the one from wheat chloroplast (Raines et al., 1988), and a complete nucleotide sequence of $c f x F$ from a prokaryote is not available. Only the sequences of the $c f x P$ genes from spinach and $A$. eutrophus have been reported thus far (Kossmann et al., 1990; Roesler \& Ogren, 1988).

It thus appears that both PRK and FBPase have received only minor attention despite their important role in the Calvin cycle. We therefore set out to characterize the cloned PRK and FBPase structural genes from Xanthobacter flavus H4-14, a bacterium capable of autotrophic growth on methanol and molecular hydrogen (Lidstrom-O'Connor et al., 1983; Meijer et $a l ., 1990 a$ ). In this paper we report the complete nucleotide sequences of these genes.

\section{Methods}

Bacterial strains and plasmids. Escherichia coli JM101 (YanischPerron et al., 1985) was used as a host for pBLuESCRIBE (Vector Cloning Systems), M13mp18 and M13mp19 (Yanisch-Perron et al., 1985) and their derivatives. pCD102 is a pVK 100 cosmid, containing a $24 \mathrm{~kb}$ chromosomal DNA fragment from $X$. flavus $\mathrm{H} 4-14$, encoding FBPase and PRK (Lehmicke \& Lidstrom, 1985, Meijer et al., 1990b).

Media and growth conditions. E. coli strains were grown on LB medium at $37^{\circ} \mathrm{C}$ (Maniatis et al., 1982). Agar was added to $1.5 \%(w / v)$ to solidify the medium. When necessary the following supplements 


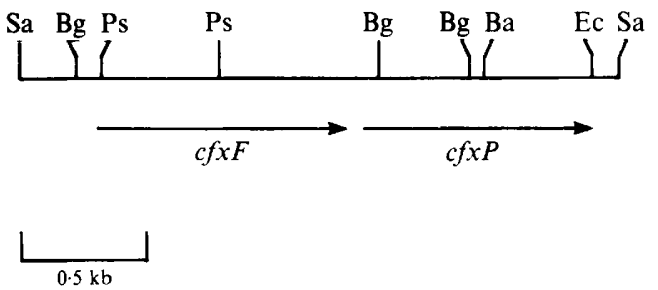

Fig. 1. Restriction map of the $2.5 \mathrm{~kb}$ DNA SalI fragment from $X$. flavus $\mathrm{H} 4-14$ and the positions of $c f x F$ and $c f x P$. Arrows indicate the direction of transcription. Restriction sites: $\mathrm{Ba}, \mathrm{BamHI} ; \mathrm{Bg}, \mathrm{Bg} / \mathrm{II}$; Ec, EcoRI; Ps, PstI; Sa, SalI.

were added (in $\mu \mathrm{g} \mathrm{ml}^{-1}$ ): ampicillin, $50 ; 5$-bromo-4-chloro-3-indolyl $\beta$ D-galactoside, 20 ; tetracycline, $12 \cdot 5$. Isopropyl $\beta$-D-thiogalactoside was used at a concentration of $0.1 \mathrm{mM}$.

DNA manipulations. Plasmid DNA was isolated from E. coli by using either the Triton X-100 lysis method of Rodriguez \& Tait (1983), in which the column chromatography step was omitted for preparative isolations, or the alkaline lysis method of Birnboim \& Doly (1979) for isolations on a smaller scale. Restriction enzymes and T4 DNA ligase were used according to the manufacturer's instructions. Analysis of restriction digests by gel electrophoresis, recovery of DNA fragments from low-melting-point agarose and transformation of $E$. coli were carried out as described by Maniatis et al. (1982). Sequence determination was done as described by Sanger et al. (1977), except that 7-deaza-dGTP was used instead of dGTP, and Sequenase (United States Biochemical Corporation) was used instead of the Klenow fragment of DNA polymerase. When necessary, custom primers (Eurosequence, Groningen, the Netherlands) were used instead of the universal primer.

\section{Results and Discussion}

\section{Nucleotide sequences of $c f x F$ and $c f x P$}

The localization of the $c f x F$ and $c f x P$ genes in $X$. flavus H4-14, and their cotranscription in the order $c f x F P$, has been established in previous work (Meijer et al., 1990b; Fig. 1). A $2.5 \mathrm{~kb} \mathrm{SalI}$ fragment from pCD102 encoding FBPase and PRK was subcloned in pBLUESCRIBE, yielding plasmid $\mathrm{pWL} 5$. This plasmid was digested with the appropriate restriction enzymes, and the restriction fragments were ligated into M13mp18 and M13mp19. The total nucleotide sequence was derived from both DNA strands and fully overlapped. The $\mathrm{C}+\mathrm{G}$ content of the SalI fragment was $66.4 \mathrm{~mol} \%$, which is close to the $\mathrm{C}+\mathrm{G}$ content of the chromosomal DNA $(68 \mathrm{~mol} \%$; Meijer et al., 1990a).

Two open reading frames (ORFA, ORFB) were identified in the nucleotide sequence, each preceded by plausible ribosome-binding sites. Both were transcribed in the same direction as $c f x F$ and $c f x P$ (Meijer et al., $1990 \mathrm{~b}$ ), and were separated by only $39 \mathrm{bp}$. ORFA had a length of $1092 \mathrm{bp}$, and would encode a protein of $38739 \mathrm{Da}$. ORFB had a length of $873 \mathrm{bp}$, encoding a protein of $33409 \mathrm{Da}$. Due to the high $\mathrm{C}+\mathrm{G}$ content, the codon usage in both ORFA and ORFB displayed a strong bias towards the use of codons having a $C$ or $G$ in the wobble position.

The deduced amino acid sequences derived from both ORFs were compared to 10856 entries in the SWPROTdatabase (Protein Database, Release 11, June 1989, University of Geneva, Switzerland), using the program FASTP (k-tuple $=2$; Lipman \& Pearson, 1985). On the basis of this comparison, ORFA and ORFB were identified as $c f x F$ and $c f x P$, respectively. The deduced molecular masses of the $c f x F$ and $c f x P$ gene products corresponded well with the sizes of the $c f x F$ gene product, expressed in $E$. coli $(40 \mathrm{kDa} ;$ W. G. Meijer \& L. Dijkhuizen, unpublished observations), and PRK from $X$. flavus H4-14 (33 kDa; Lehmicke \& Lidstrom, 1985). The nucleotide sequences and deduced amino acid sequences of $c f x F$ and $c f x P$ are shown in Fig. 2.

\section{Comparison of FBPase primary structures}

A comparison of the primary structures of FBPase from various sources is shown in Fig. 3. The FBPase amino acid sequence from $X$. flavus $\mathrm{H} 4-14$ displays 32 to $38 \%$ similarity with FBPases from heterotrophic sources, which show 41 to $63 \%$ similarity amongst each other (Table 1). Of the two autotrophic FBPase proteins, from wheat chloroplasts and $A$. eutrophus, only the latter shows a high similarity $(52 \%)$ with the $X$. flavus H4-14 protein, although all three autotrophic FBPase proteins have the same physiological role. In contrast, the $\mathrm{RuBisC} / \mathrm{O}$ proteins from plants are very similar to those from $A$. eutrophus and X. flavus H4-14 (Andersen \& Caton, 1987; Meijer et al., 1990b). An explanation for this difference in sequence conservation could be that the structural constraints in $\mathrm{RuBisC} / \mathrm{O}$, a hexadecamer consisting of two types of subunits, are higher than those in the FBPase protein, allowing less sequence variation. Alternatively, the FBPase proteins from eukaryotic and prokaryotic species may have evolved from different ancestors.

Recently the three-dimensional structure of pig kidney FBPase and its complexes with AMP and fructose 2,6bisphosphate (F-2,6-P) have become available ( $\mathrm{Ke}$ et al., 1989). AMP and F-2,6-P inhibit FBPase activity in a number of organisms. AMP inhibition is allosteric, whereas F-2,6-P probably binds to the active site ( $\mathrm{Ke} e t$ al., 1989; Liu et al., 1989). The F-2,6-P-binding site consists of the following residues (numbering for pig kidney FBPase): Asn-212, Tyr-244, Gly-246, Ser-247, Met-248, Tyr-264 and Lys-274. These residues are either identical, or represented by a conservative replacement in the $X$. flavus H4-14 protein. Residues making up a negatively charged pocket, forming one wall of the 
-

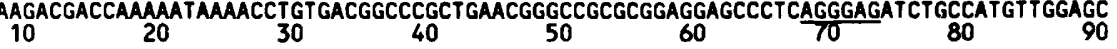
N A D H R A A V A O A A G VAA A R I T L T V M L D E HA G CGAACGCAGACCATCGGGCCGCAGTTGCCCAGGCTGCAGGTGTCGCAGCCTCGCGTATCACGCTCACCGTCATGCTCGACGAGTGGGCGG
100

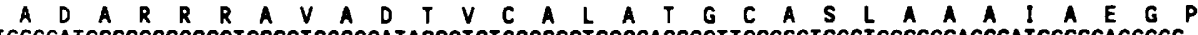
GTGCGGATGCCCGCCGCCGTGCCGTCGCGGATACCGTCTGCGCCCTCGCCACCGGTTGCGCGTCGCTGGCCGCAGCCATCGCCGAGGGGC L A G D L A R T L S S G E A G E G O K A L D V I S N D I $V$ I

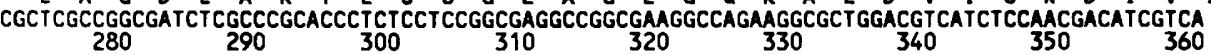

G A L K A A P V A A V A S E E N D A P V L L D P T A P L L V TCGGCGCGCTGAAGGCGGCGCCGGTCGCGGCGGTCGCATCCGAGGAGAACGACGCCCCGGTCCTGCTCGATCCCACCGCGCCGCTGCTCG

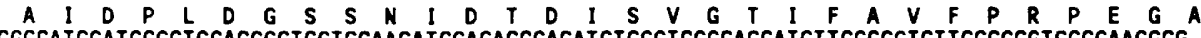

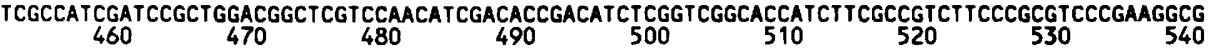

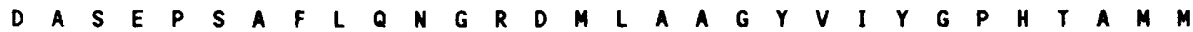
CCGACGCGTCCGAACCCTCTGCCTTCCTGCAGAACGGGCGGGACATGCTGGCCGCCGGITATGTGATCTACGGCCCCCACACCGCGATGA

L T L G A G T H H F A L D R A G L F R L V D A E V K V K E G $\underset{640}{\operatorname{TSCTGACGTCGCGCCGGCACCTGGCACTTCGCCCTCGACCGCGCCGGCCTCTTCCGCCTGGTCGATGCAGAGGTGAGGTGAAGGAGG~}}$

A A E F A I N M S $N$ N Y

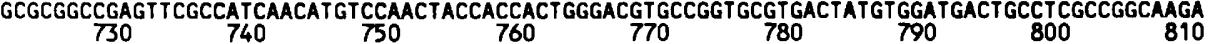

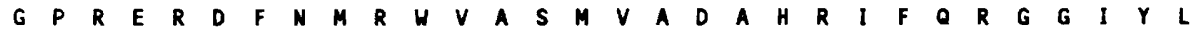
AGGGGCCGCGGGAGCGCGACTTCAACATGCGCTGGGTGGCCTCCATGGTGGCGGACGCGCATCGCATCTTCCAGCGCGGCGGCATCTATC

Y P P G D G R K G Y T TCTATCCGGGCGATGGCCGGAAGGGCTACACGCACGGCCGCCTGCGCCICCICTACGAGGCCTICCCCGTCGCCTTCCIGATGGAGCAGG

S G S A T D G R G A I L D L S A T G L H Q R V P F I F G S R

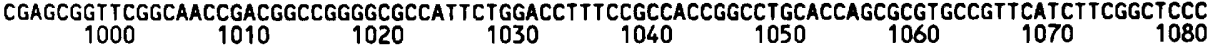

D E E V V A A R V V S S R Y Y GCGACGAGGTGGCCCGGGTCTCCCGCTATCACCTGGAGCCGAACGGCCATGGCGAGCGCTCGCCGCTGTTCGCGCGGCGCGGACTGTICA

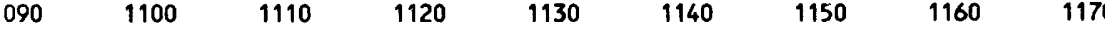

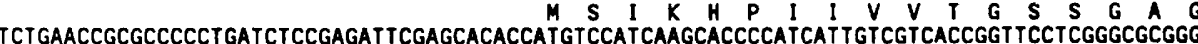
$118001190 \quad 1200 \quad 1210 \quad 1220 \quad 1230 \quad 1240 \quad 1250 \quad 1260$

T $T$ S S V V

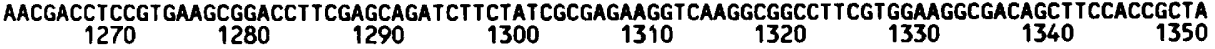

D R Y Y E M R E L M A A E A A K G N K H F S $H A$ F S P P E T N R L CGACCGCTATGAGATGCGCGAGCTGATGGCCGCCGAGGCGGCCAAGGGCAACAAGCACTTCAGCCACTTCTCGCCCGAGACCAACCGGCT

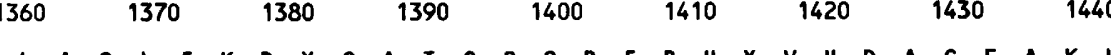

D D L A O L F K D Y G A T G S G R F R H Y V H D A G E A A K L

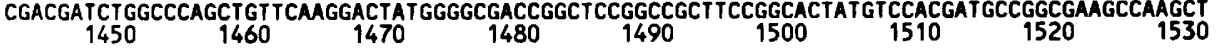

Y N $N$ T E P P G R F T D W E D L E O G T D I L F Y E G L H G A V

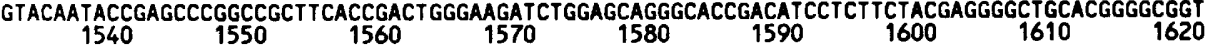

$V$ T D CGTCACCGACGAACTGAACCTCGCCCAGCATGCCGACCTGAAGATCGGCGTGGTGCCCGTGATCAACCTGGAGTGGATCCAGAAGATCCA

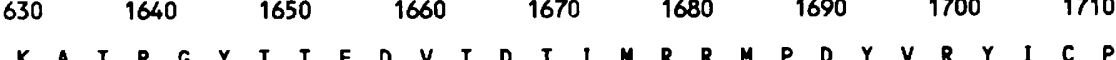

R D K A T R G Y T T T E $17201730 \quad 1740 \quad 1750 \quad 1760 \quad 1770 \quad 1780 \quad 1790 \quad 1800$

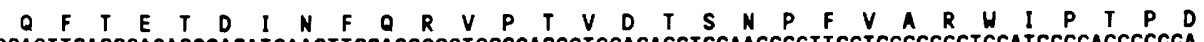

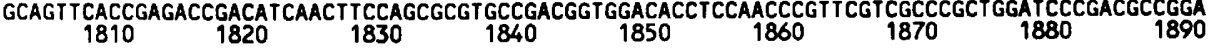

E S M V V V I I R F F R D D P A CGAATCGATGGTCGTGATCCGCTTCCGCGACCCGCACGGCATCGATTTCCCCTATTTGCTGTCGATGATCCACAACAGCTTCATGTCGCG

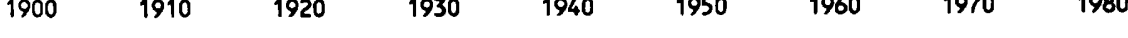

A N S I V I P G M K O D L A M O L L L T P L I M K L M D R K

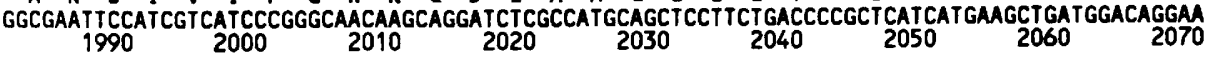

$R \quad R \quad A$ GCGCCGAGCCGGCTGAGCCGGGCGCGAAGCTCCC
Fig. 2. Nucleotide sequence and deduced amino acid sequence of $c f x F$ and $c f x P$ from $X$. flavus $\mathrm{H} 4-14$. The first open reading frame represents $c f x F$ and the second $c f x P$. The amino acids are represented by the oneletter code. The putative ribosomebinding sites are underlined. 

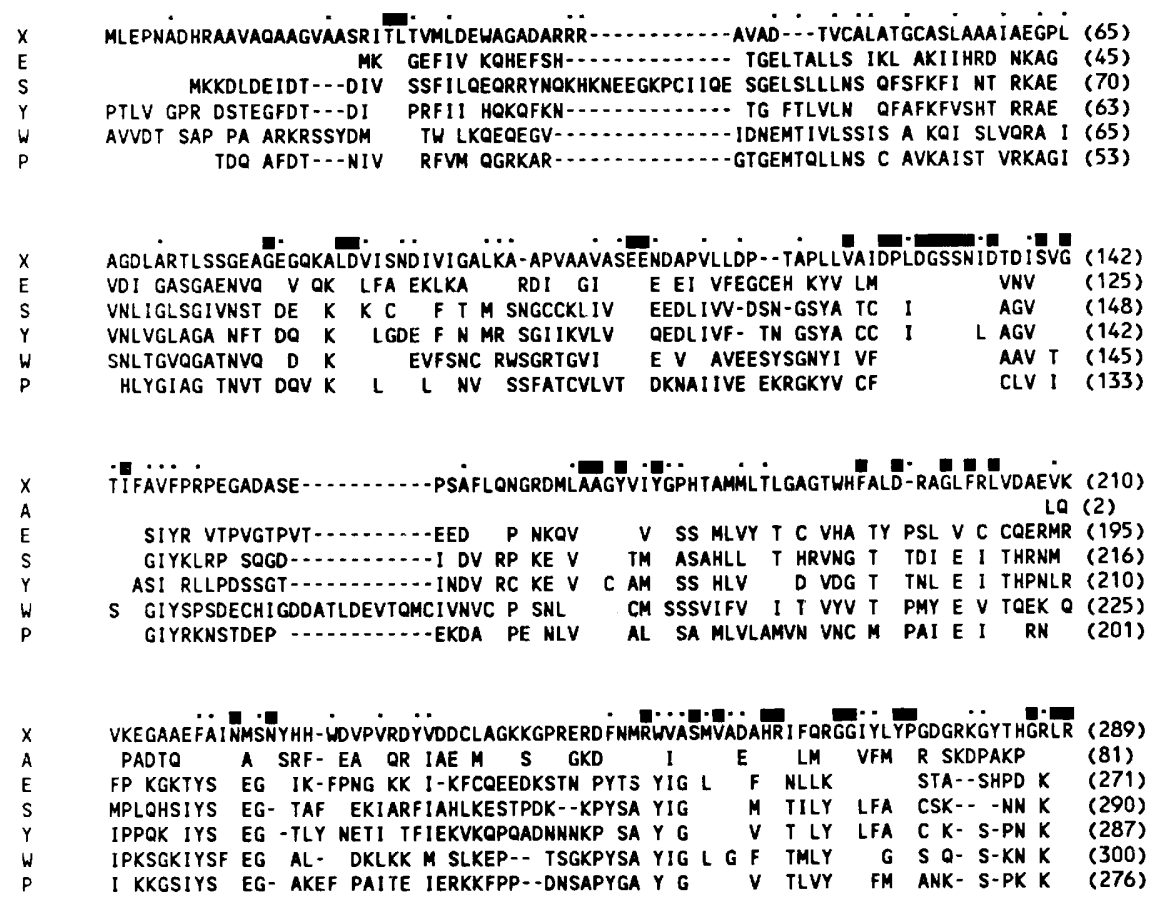

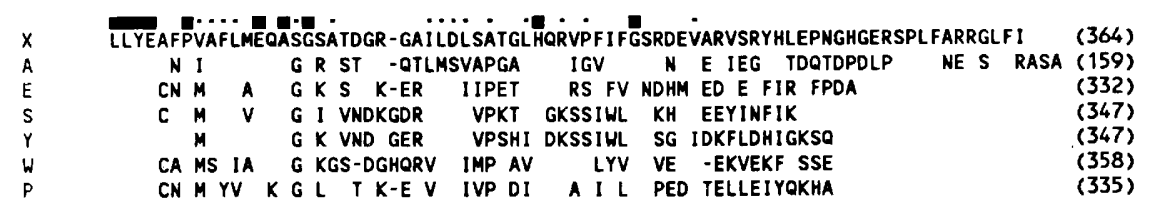

Fig. 3. Alignment of FBPase proteins from: X, X. flavus H4-14; A, Alcaligenes eutrophus (Kossmann et al., 1990); E, Escherichia coli (Hamilton et al., 1988); S, Schizosaccharomyces pombe (Rogers et al., 1988); Y, Saccharomyces cerevisiae (Rogers et al., 1988); W, wheat chloroplast (Raines et al., 1988); P, pig kidney (Marcus et al., 1982). The mature form of the chloroplast FBPase was used in the alignment. $\mathbf{\square}$, identical residue; ., conservative substitution following the scheme: PAGST, QNED, ILVM, HKR, YFW, C. Only similarities between more than two sequences are indicated. Numbers in parentheses refer to amino acids. Except for the terminal residues, only the residues that differ from the $X$. flavus $\mathrm{H} 4-14$ sequence are indicated.

Table 1. Similarity between FBPase primary structures

Similarities are in percentages. X, Xanthobacter flavus H4-14; A, carboxy terminus from Alcaligenes eutrophus (Kossmann et al., 1990); E, Escherichia coli (Hamilton et al., 1988); W, wheat chloroplast (Raines et al., 1988); P, pig kidney (Marcus et al., 1982); Y, Saccharomyces cerevisiae (Rogers et al., 1988); S, Schizosaccharomyces pombe (Rogers et al., 1988).

\begin{tabular}{lrrrrrrr} 
& \multicolumn{1}{c}{ X } & A & E & W & P & Y & S \\
X & 100 & & & & & & \\
A & 52 & 100 & & & & & \\
E & 38 & 38 & 100 & & & & \\
W & 34 & 39 & 46 & 100 & & & \\
P & 36 & 36 & 45 & 46 & 100 & & \\
Y & 35 & 36 & 43 & 41 & 47 & 100 & \\
S & 32 & 40 & 46 & 43 & 46 & 63 & 100
\end{tabular}

F-2,6-P-binding site, Asp-118, Asp-121, Glu-280, Glu97, Glu-98, Lys-71 and Arg-276 (numbering for pig kidney FBPase) are identical in the $X$. flavus H4-14 FBPase sequence. $\mathrm{Mg}^{2+}$, which is essential for FBPase activity, interacts with both the negatively charged pocket and a phosphate group from F-2,6-P. This strongly suggests that $\mathrm{F}-2,6-\mathrm{P}$ binds to the active site ( $\mathrm{Ke}$ et al., 1989). Residues close to the AMP-binding site in the pig FBPase sequence are either not conserved or are represented by a conservative substitution in the $X$. flavus $\mathrm{H} 4-14$ sequence.

The fact that the F-2,6-P-binding site residues are strongly conserved in the $X$. flavus H4-14 FBPase sequence indicates that this region is indeed essential for FBPase activity, and thus may constitute the active site. Residues close to the AMP-binding site are not conserved. The Nocardia opaca FBPase, which is specifically induced during autotrophic growth, is not very sensitive to AMP. In contrast, the FBPase isoenzyme functioning in gluconeogenesis in the same organism is fully inhibited by AMP at concentrations of $100 \mu \mathrm{M}$ (Amachi $\&$ Bowien, 1979). The properties of the enzyme in $X$. flavus H4-14 are currently under investigation.

Characteristics which are specific for a particular FBPase, such as the insertions in the Schizosaccharomyces pombe and wheat chloroplast FBPase sequences, 


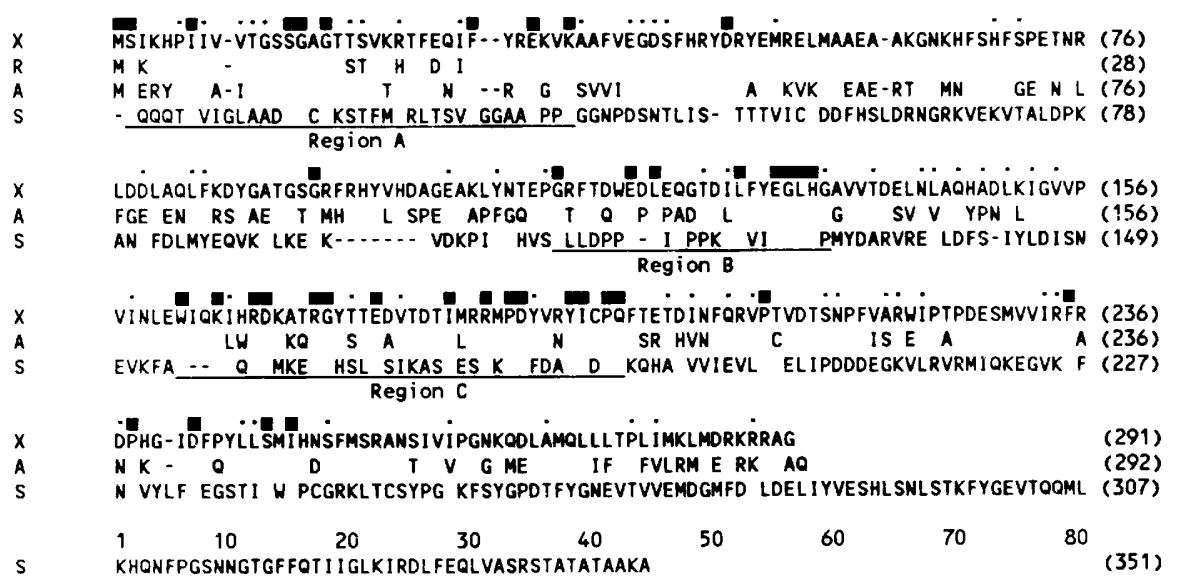

Fig. 4. Alignment of PRK proteins from: X, X. flavus H4-14; A, Alcaligenes eutrophus (Kossmann et al., 1990); R, Rhodobacter sphaeroides (Hallenbeck \& Kaplan, 1987); S, spinach (Roesler \& Ogren, 1988). $\square$, identical residue; ., conservative substitution as in Fig. 3. Only similarities between more than two sequences are indicated. Regions of identity are underlined (see text). Numbers in parentheses refer to amino acids. Except for the terminal residues, only the residues that differ from the $X$. flavus $\mathrm{H} 4-14$ sequence are indicated.

and the cAMP-dependent protein kinase consensus sequence in the Saccharomyces cerevisiae amino terminus, are not conserved in the $X$. flavus H4-14 sequence (Fig. 3; Raines et al., 1988; Rogers et al., 1988).

\section{Comparison of $P R K$ primary structures}

As was observed for the FBPase protein, the PRK amino acid sequence from $X$. flavus $\mathrm{H} 4-14$ is not very similar (less than $22 \%$ identity) to that from a eukaryotic plant, in this case spinach (Fig. 4). In contrast, the $X$. flavus H4-14 and $A$. eutrophus proteins display $65 \%$ similarity. This does not come as a surprise, as the quaternary structure of prokaryotic and eukaryotic PRK proteins is very different. In bacteria, PRK is present as an octamer of $33 \mathrm{kDa}$ subunits, whereas in spinach, PRK is a dimer of $45 \mathrm{kDa}$ subunits (Krieger \& Miziorko, 1986; Rippel \& Bowien, 1984; Siebert \& Bowien, 1984). This indicates that residues important in subunit interaction in the prokaryotic holoenzyme are not conserved in the eukaryotic proteins.

In the amino-terminal protein sequence of PRK of both prokaryotic and eukaryotic origin a sequence can be recognized which is supposed to be an ATP-binding site (region A, Fig. 4; Hallenbeck \& Kaplan, 1987; Klintworth et al., 1985; Porter et al., 1988). The spinach PRK sequence contains two unique cysteine residues, one at position 16, within the proposed ATP-binding site consensus sequence, the other at position 55 . These cysteine residues can be oxidized to form a disulphide bridge, causing inactivation of PRK (Porter et al., 1988). The oxidation and alkylation of Cys- 16 by (bromoacetyl) ethanolamine phosphate can be prevented by ATP, strengthening the hypothesis that Cys-16 is at the active site (Porter \& Hartman, 1986). The functions of the other conserved regions (B and $\mathrm{C}$ : Fig. 4) remain to be established, although they may play a role in the binding of the substrate, RuMP. The identified conserved regions may be suitable targets for mutagenesis to elucidate their function.

The authors thank Dr B. Bowien for making results available prior to* publication, and Professor Dr W. Harder for valuable discussions. The investigations were supported by the Foundation for Fundamental Biological Research (BION), which is subsidized by the Netherlands Scientific Organization (NWO).

\section{References}

AMACHI, T. \& BowIEN, B. (1979). Characterization of two fructose bisphosphatase isoenzymes from the hydrogen bacterium Nocardia opaca 1b. Journal of General Microbiology 113, 347-356.

ANDERSEN, K. \& CATON, J. (1987). Sequence analysis of the Alcaligenes eutrophus chromosomally encoded ribulose bisphosphate carboxylase large and small subunit and their gene products. Journal of Bacteriology 169, 4547-4558.

BIRNBoIM, H. C. \& Doly, J. (1979). A rapid alkaline extraction procedure for screening of recombinant plasmid DNA. Nucleic Acids Research 7, 1513-1523.

GiBson, J. L. \& TABITA, F. R. (1987). Organization of phosphoribulokinase and ribulose bisphosphatase carboxylase/oxygenase genes in Rhodopseudomonas (Rhodobacter) sphaeroides. Journal of Bacteriology 169, 3685-3690.

GiBson, J. L. \& TABITA, F. R. (1988). Localization and mapping of $\mathrm{CO}_{2}$ fixation genes within two gene clusters in Rhodobacter sphaeroides. Journal of Bacteriology 170, 2153-2158.

Hallenbeck, P. L. \& KaPLAN, S. (1987). Cloning of the gene for phosphoribulokinase activity from Rhodobacter sphaeroides and its expression in Escherichia coli. Journal of Bacteriology 169, 3669-3678.

Hamilton, W. D. O., Harrison, D. A. \& Dyer, T. A. (1988). Sequence of the Escherichia coli fructose-1,6-bisphosphatase gene. Nucleic Acids Research 16, 8707. 
Ke, H., Thorpe, C. H., Seaton, B. A., Marcus, F. \& Lipscomb, W. N. (1989). Molecular structure of fructose-1,6-bisphosphate at $2 \cdot 8-\AA$ resolution. Proceedings of the National Academy of Sciences of the United States of America 86, 1475-1479.

Klintworth, R., HusemanN, M., Salnikow, J. \& Bowien, B. (1985). Chromosomal and plasmid locations for phosphoribulokinase genes in Alcaligenes eutrophus. Journal of Bacteriology 164, 954-956.

Klintworth, R., Husemann, M., Weissenborn, C. \& Bowien, B. (1988). Expression of the plasmid-encoded phosphoribulokinase gene from Alcaligenes eutrophus. FEMS Microbiology Letters 49, $1-6$.

Kossmann, J., Klintworth, R. \& Bowien, B. (1990). Sequence analysis of the chromosomal and plasmid genes encoding phosphoribulokinase from Alcaligenes eutrophus. Gene 85, 247-252.

KrIEGER, T. J. \& Miziorko, H. M. (1986). Affinity labelling and purification of spinach leaf ribulose-5-phosphate kinase. Biochemistry 25, 3496-3501.

LeHMicKe, L. G. \& LiDSTROM, M. E. (1985). Organization of the genes necessary for growth of the hydrogen-methanol autotroph Xanthobacter sp. strain H4-14 on hydrogen and carbon dioxide. Journal of Bacteriology 162, 1244-1249.

Lidstrom-O'Connor, M. E., Fulton, G. L. \& Wopat, A. E. (1983). Methylobacterium ethanolicum: a syntrophic association of two methylotrophic bacteria. Journal of General Microbiology 129, 31393148 .

Lipman, D. J. \& Pearson, W. R. (1985). Rapid and sensitive protein similarity searches. Science 227, 1435-1441.

LiU, F., RoY, M. \& Fromm, H. J. (1989). The site of substrate and fructose 2,6-bisphosphate binding to rabbit liver fructose-1,6bisphosphatase. Biochemical and Biophysical Research Communications 161, 689-695.

Maniatis, T., Fritsch, E. F. \& Sambrook, J. (1982). Molecular Cloning: a Laboratory Manual. Cold Spring Harbor, NY: Cold Spring Harbor Laboratory.

Marcus, F., Edelstein, I., Reardon, I. \& Heinrikson, R. L. (1982). Complete amino acid sequence of pig kidney fructose-1,6-bisphosphatase. Proceedings of the National Academy of Sciences of the United States of America 79, 7161-7165.

Meijer, W. G., Croes, L. M., Jenni, B., Lehmicke, L. G., Lidstrom, M. E. \& DiJKHUIZEN, L. (1990a). Characterization of Xanthobacter strains H4-14 and 25a and enzyme profiles after growth under autotrophic and heterotrophic conditions. Archives of Microbiology 153, 360-367.

MeiJer, W. G., Arnberg, A. C., Enequist, H. G., Terpstra, P., LidSTROM, M. E. \& DiJkhuizen, L. (1990b). Identification and organization of carbon dioxide fixation genes in Xanthobacter flavus H4-14. Molecular and General Genetics (in the Press).

Porter, M. A. \& Hartman, F. C. (1986). Commonality of catalytic and regulatory sites of spinach phosphoribulokinase: characterization of a tryptic peptide that contains an essential cysteinyl residue. Biochemistry 25, 7314-7318.

Porter, M. A., Stringer, C. D. \& Hartman, F. C. (1988). Characterization of the regulatory thioredoxin site of phosphoribulokinase. Journal of Biological Chemistry 263, 123-129.

Raines, C. A., Lloyd, J. C., Longstaff, M., Bradley, D. \& Dyer, T. A. (1988). Chloroplast fructose-1,6-bisphosphatase: the product of a mosaic gene. Nucleic Acids Research 16, 7931-7942.

RIPPEL, S. \& BowIEN, B. (1984). Phosphoribulokinase from Rhodopseudomonas acidophila. Archives of Microbiology 139, 207-212.

RoDRIGUEZ, R. L. \& TAIT, R. C. (1983). Recombinant DNA Techniques: an Introduction. Reading, Massachusetts: Addison-Wesley.

ROESLER, K. R. \& OGREN, W. L. (1988). Nucleotide sequence of spinach cDNA encoding phosphoribulokinase. Nucleic Acids Research 16, 7192.

Rogers, D. T., Hiller, E., MrTsock, L. \& ORR, E. (1988). Characterization of the gene for fructose-1,6-bisphosphatase from Saccharomyces cerevisiae and Schizosaccharomyces pombe. Journal of Biological Chemistry 263, 6051-6057.

SANGer, F., Nicklen, S. \& Coulson, A. R. (1977). DNA sequencing with chain terminating inhibitors. Proceedings of the National Academy of Sciences of the United States of America 74, 5463-5467.

SIEBERT, K. \& BowIEN, B. (1984). Evidence for an octameric structure of phosphoribulokinase from Alcaligenes eutrophus. Biochimica et Biophysica Acta 787, 208-214.

TABITA, F. R. (1988). Molecular and cellular regulation of autotrophic carbon dioxide fixation in microorganisms. Microbiological Reviews 52, 155-189.

YanisCh-Perron, C., Vieira, J. \& Messing, J. (1985). Improved M13 phage cloning vectors and host strains. Nucleotide sequences of the M13mp18 and pUC19 vectors. Gene 33, 103-119. 